M. Vanier

E. Dutil

J. Provost

J. Lambert

J.M. Mazaux

J. Sullivan

D. Melanson

N. Chadan

A. Forget

Y.L. Boulanger

\section{ADRESSES}

M. Vanier, E. Dutil : Centre de recherche, Institut de réadaptation de Montréal. École de réadaptation, U. de Montréal. J. Provost : Centre de recherche, Institut de réadaptation de Montréal, Département des sciences neurologiques, hôpital du SacréCœur de Montréal. J. Lambert : Département de médecine sociale et préventive, U de Montréal. J.M. Mazaux : Service de rééducation fonctionnelle neurologique, $\mathrm{CHU}$ de Bordeaux. J. Sullivan : Centre de recherche, Institut de réadataption de Montréal, département des sciences de l'exercice, U. Concordia. D. Melanson; département de radiologie, Institut neurologique de Montréal. N. Chadan; Service de neurochimie, CHU de Dijon. A. Forget : École de réadaptation, U. de Montréal. Y.L. Boulanger: Centre de recherche, Institut de réadaptation de Montréal, département de

\title{
Les séquelles psychologiques des traumatismes crâniens graves et leurs répercussions sur l'autonomie sociale et professionnelle des victimes
}

Les traumatismes crâniens constituent une cause majeure d'handicaps à long terme. Ceux-ci sont en grande partie associés aux séquelles psychologiques multiples de l'atteinte encéphalique. Les travaux récents ont fourni une description des processus neuropathologiques en cause ainsi qu'une meilleure compréhension des principales séquelles psychologiques d'un traumatisme crânien grave et leurs répercussions sur l'autonomie des personnes qui en sont atteintes.

\section{Introduction}

Les traumatismes crâniens constituent une cause majeure d'handicaps à long terme. Ces handicaps sont en grande partie associés aux séquelles psychologiques multiples de l'atteinte encéphalique. Depuis une quinzaine d'années, des équipes de recherche ont entrepris des études approfondies sur les séquelles des traumatismes crâniens et sur leurs répercussions sur les activités sociales et professionnelles des victimes.

Nous aborderons dans cet article les processus neuropathologiques en cause, ainsi que les principales séquelles psychologiques d'un traumatisme crânien grave et leurs répercussions sur l'autonomie des personnes qui en sont atteintes. Nous ne présenterons pas les séquelles neurophysiologiques comme les troubles neuro-orthopédiques, les troubles périphériques et les troubles neuromoteurs centraux (la lenteur, les troubles de précision des gestes, les dystonie, les ataxies et les troubles d'équilibre, etc.)

\section{Quelques données épidémiologiques}

Au Québec [1], un décès sur 12 survient à la suite d'un accident. Les traumatismes constituent la troisième cause de décès tous âges confondus. Ils représentent la première cause de décès des personnes de moins de 46 ans. Pour chaque perte de vie par traumatisme, on dénombre 16 cas d'hospitalisation et 26 personnes obligées de restreindre leurs activités, ce qui met en évidence l'importance du taux de morbidité par rapport au taux de mortalité. Par ailleurs, sur les 4000 personnes hospitalisées annuellement pour un traumatisme crânio- 
encéphalique, $15 \%$ ont une atteinte encéphalique dont la gravité s'étend de modérée à très grave [2]. Malgré les progrès importants réalisés par la médecine d'urgence, le pronostic à long terme des traumatismes graves demeure sombre; plus de la moitié des victimes ont moins de trente ans, et bien que la majorité d'entre elles regagnent leur domicile deux ans après avoir subi le traumatisme, rares sont celles qui reprennent une activité productive significative (travail, études, responsabilités au foyer). Les données françaises et américaines sont tout aussi alarmantes [3, 4]. Toutes les études soulignent la nécessité, d'une part, d'augmenter les mesures de prévention des traumatismes et d'autre part, de développer des interventions appropriées à cette problématique.

\section{Les mécanismes du traumatisme crânien et les principales neuropathologies}

Deux phénomènes mécaniques principaux sont responsables des dommages à l'encéphale : les phénomènes liés au contact et ceux liés à la force d'inertie (accélération) [5]. Le point de contact peut être le siège de fractures du crâne et de contusions ou de lacérations cérébrales (contusions de " coup "). La force d'inertie, et davantage l'accélération rotatoire que l'accélération linéaire, peut causer des déformations ou des tensions cérébrales (décrites selon la direction de la force : déchirements, compressions, cisaillements), causes directes de certaines affections primaires, dont les lésions axonales diffuses, les contusions focalisées et les hématomes sous-duraux [6]. L'importance des conséquences dépend aussi de la vitesse et de la durée d'accélération [7] : la plupart du temps, la déformation produite à basse vitesse endommage peu les tissus; à haute vitesse elle brise leur viscoélasticité et provoque des dommages importants ; une accélération de longue durée entraîne des dommages tissulaires plus importants encore.

Les dommages causés à l'encéphale sont habituellement présentés selon les catégories suivantes : les pathologies primaires provoquées par $m / s n^{\circ} 5$ vol. 9 , mai 93 l'impact et les pathologies secondaires, c'est-à-dire consécutives à cellesci, les pathologies extra-cérébrales et intracérébrales et, enfin, les pathologies précoces et tardives, auxquelles il faut ajouter les pathologies circonscrites et les pathologies diffuses : contusions circonscrites, lésions axonales diffuses et lésions hypoxiquesischémiques. Les atteintes seront présentées selon la dichotomie lésions circonscrites - lésions diffuses.

Les contusions, les hémorragies des noyaux gris centraux et les lésions hypoxiques-ischémiques font partie des pathologies primaires circonscrites de l'encéphale. Les contusions se produisent de la surface corticale vers les structures profondes, le plus souvent bilatéralement, dans les parties antérieures et inférieures des lobes frontaux et temporaux, le cerveau ayant heurté certaines surfaces crâniennes irrégulières de ces régions lors de son déplacement. La dimension et la profondeur des contusions déterminent fortement l'évolution clinique. Les hémorragies des noyaux gris centraux se produisent à la suite de ruptures d'artères pénétrantes, comme les artères lenticulostriées et choroïdales antérieures. Les lésions hypoxiques-ischémiques circonscrites, résultent le plus souvent de l'infarcissement d'un territoire vasculaire dû à une compression ou à un déplacement ; l'artère cérébrale postérieure est particulièrement sujette à une compression résultant d'une hernie transtentorielle du lobe temporal.

Les pathologies primaires diffuses regroupent les lésions axonales diffuses et les lésions hypoxiquesischémiques diffuses. Les premières sont causées par une tension exercée sur les axones; cette tension peut provoquer par la suite la destruction de ces axones et conduire à une atrophie généralisée ; ce type de lésion se produit de la périphérie vers les régions profondes, touchant, dans les cas les plus sévères, le corps calleux et le mésencéphale dorsolatéral. L'augmentation des taux de neurotransmetteurs comme le glutamate, l'aspartate et l'acetylcholine provoquent des phénomènes d'autodestruction au sein des cellules; certaines structures possédant un grand nombre de récepteurs à ces transmetteurs sont particulièrement susceptibles d'être touchées, en particulier l'hippocampe [8], ce qui les rend très vulnérables à d'autres pathologies comme l'hypoxie [9, in 5]. Le diagnostic de lésions axonales diffuses est principalement établi à partir de l'histoire et de l'évolution cliniques et des images de tomodensitométrie et de résonance magnétique : une histoire de traumatisme mettant en jeu des mécanismes d'accélération importants (accident de la route, chute de plus de deux mètres) à laquelle sont associées une perte de conscience immé diate sans intervalle de lucidité et des images cérébrales montrant, en phase aiguë, un œdème diffus, avec des hémorragies ponctiformes et de petites hémorragies sous-arachnoïdiennes et intraventriculaires. Les indices actuellement considérés pour déterminer la gravité des lésions sont la profondeur initiale du coma et sa durée, ainsi que la durée de l'amnésie posttraumatique (confusion post-coma) [5].

Des complications cardiovasculaires ou respiratoires sont généralement à l'origine de l'hypoxie-ischémie diffuse fréquemment associée à des périodes prolongées d'hypertension intracrânienne grave. L'hypoxie-ischémie se manifeste par une perte diffuse de neurones corticaux, par des infarcissements des zones limitrophes des territoires vasculaires et par l'atteinte de régions particulièrement vulnérables à l'hypoxie comme l'hippocampe et les noyaux gris centraux [5]. Cette pathologie accompagne fréquemment les lésions axonales diffuses; elle s'en distingue sur la base d'une histoire d'hypoxie ou d'hypertension intracrânienne grave, d'une évolution clinique s'éloignant de celle des lésions axonales diffuses et de signes cliniques compatibles avec la distribution de la pathologie ; l'imagerie cérébrale n'est utile que dans les cas où de petites zones d'infarctus sont visibles [5].

De nombreuses autres complications précoces ou tardives s'observent suite à un traumatisme grave; parmi les complications précoces, on remarque une compression et une hémorragie au niveau de l'hypothalamus, du tronc cérébral et des nerfs crâniens ; parmi les complications tardives, on relève l'hydrocéphalie, l'hématome sous-dural chronique et l'hygroma 


\section{RÉFÉRENCES}

1. Robitaille $Y$, Choinière R, Camirand F Les traumatismes au Québec: Leur importance sur le plan de la mortalité, de l'hospitalisation et de l'incapacité. Dans : Les publications du Québec, eds. Traumatismes comprendre pour prévenir, Québec: Ginette Beaulne, 21-66.

2. Giroux M. Le programme de réadaptation des victimes d'un traumatisme crâniocérébral suite à un accident de la route à la Société de l'assurance automobile du Québec. Actes du colloque "Traumatisme crânien : réinsertion socio-professionnelle ", 1991 ; 18-19 nov., Hôpital Juif de Réadaptation, Laval, Québec.

3. Mazaux J-M. Approche épidémiologique des traumatismes crâniens. I Réadapt Med 1985 ; vol. 5-6 : 185-9.

4. Harrison CL, Dijkers M. Traumatic brain in jury registries in the United States : an overview. Brain injury 1992 ; vol. 6, 3 203-12.

5. Katz DI, Neuropathology and neurobehavioral recovery from closed head injury. $J$ Head Trauma Rehabil $1992 ; 7$ (2) : 1-15

6. Adams JH, Gennarelli TA, Graham DI, Scoot G, Thibault LE. Diffuse axonal injury in non-missile head injury. In : Advances in Villani R, Papo I, Giovanelli M, Gaini SM and Tomei G eds. Neurotraumatology. Amsterdam : Excerpta Medica, 1983.

7. Viano DC. Biomechanics of head in jury - toward a theory linking head dynamic motion, brain tissue deformation, and neu ral trauma. Technical paper, Warrendale, Penn : Society of Automotive Engineers, 1988

8. Hayes RL, Lyeth BG, Jenkins LW Neurochemical mechanisms of mild and moderate head injury: implications for treatment. In : HS Levin, HM Eisengerb, Oxford University Press, 1989

9. Jenkins LW, Lewett W, Moszynski K, et al. Increased cerebral ischemia : the use of a controlled second insult as a research tool. Soc Neurosci Abstr. $1987 ; 13: 153$

10. Bachman DL. The diagnosis and management of common neurologie sequelae of closed head injury. J Head Trauma Rehab, june, 1992

11. Teasdale G, Teasdale E, Hadley D. Computed tomographic and magnetic resonnance imaging classification of head injury. J Neur 1992 ; vol. 9, suppl. 1 : 249-57.

12. Van Zomeren AH, Brouwer W. Attentional deficits after closed head injury. In Deelman BG, Saan RJ and van Zomeren $\mathrm{AH}$ eds. Traumatic brain injury: Clinical, social and rehabilitational aspects. Amsterdam/Lisse : Sweta \& Zeitlinger, 1990.
[10]. Selon Teasdale et coll. [11], l'imagerie par résonance magnétique constitue le meilleur moyen de visualiser les lésions associées aux incapacités à long terme. A la figure 1, sont présentées des images cérébrales par résonance magnétique réalisées trois ans après un traumatisme crânien grave. Elles montrent des lésions post-traumatiques typiques difficilement visibles à la tomodensitométrie cérébrale.

Les séquelles psychologiques de l'atteinte encéphalique, à la fois diffuse et multifocale avec prédominance aux pôles cérébraux et aux régions médianes, sont multiples ; elles con-



Figure 1. Imáges cérébrales par résonance magnétique. (A) Densité de proton. L'image montre des hyperdensités périventriculaires surtout à gauche, laissant supposant des processus de gliose. (B) Pondération T1. L'image montre une petite hypodensité dans le genu du corps calleux. (P. suivante). cernent surtout la régulation des états internes (l'état d'éveil, les besoins physiologiques complexes, comme la faim et les comportements sexuels, et les états émotionnels) et la régulation de l'activité consciente. Les répercussions sur l'autonomie sociale et professionnelle affectent toutes les sphères d'activités.

\section{Les séquelles psychologiques tardives les plus fréquentes}

Régulation de l'état d'éveil, des besoins physiologiques complexes et des états émotionnels 
Depuis le début du siècle, les descriptions cliniques consacrécs aux victimes d'un traumatisme crânioencéphalique mentionnent la présence chez ces patients d'une "lenteur de pensée ". Ce symptôme, maintenant appelé " ralentissement du traitement de l'information " sc manifeste dans les tâches d'attention sélective et d'attention divisée [12], dans les tâches qui supposent des traitements d'information et des prises de décision rapides ainsi que dans les situations d'apprentissage où la quantité de matériel à mémoriser est grande et nécessite aussi un traitement rapide. Aux tests mnésiques, aucune classe de tâches sollicitant la mémoire épisodique (événements personnels ou publics nouveaux, connaissances nouvelles n'est épargnée [13] alors que la mémoire sémantique (connaissances anciennes) demeure préservée. Les quelques données disponibles sur la mémoire prospective (par exemple, se rappeler de ce qu'on a prévu de faire) et la mémoire procédurale (développer de nouvelles habiletés comme apprendre un sport ou une tâche de travail) indiquent que ces fonctions mnésiques sont perturbées. On observe aussi des changements dans l'organisation des comportements en relation avec les besoins physiologiques et les instincts (appétit, comportement défensif, libido)

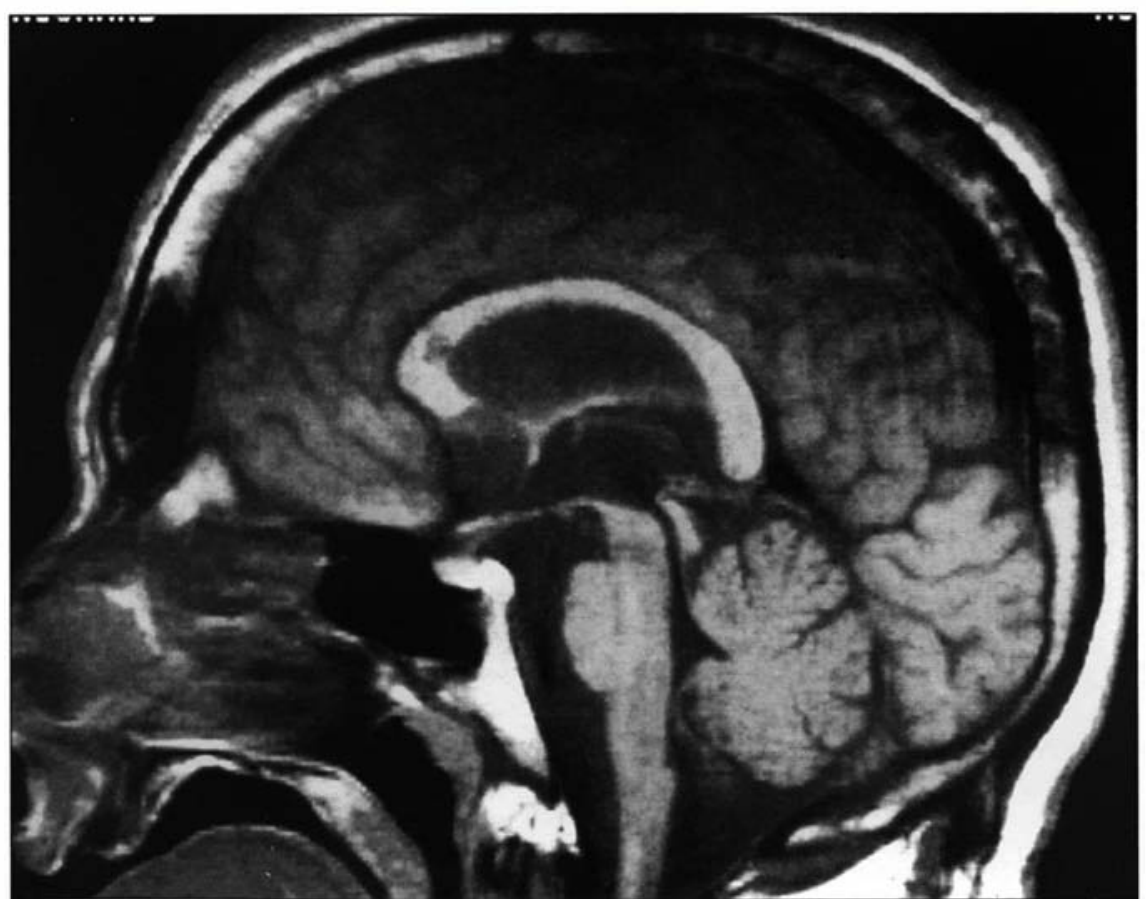

[14], une diminution des intérêts, des perturbations de l'intensité et de la qualité des sentiments, de la durée et de la stabilité des états affectifs et des perturbations de type émoussement des affects (apathie, indifférence générale), irritabilité, incongruité de l'affect, labilité émotionnelle, anxiété, dépression et, parfois, excitation se manifestant dans le comportement moteur ou dans l'humeur. Une tendance à une lassitude rapide (fatigabilité) est fréquemment notée [15].

\section{Régulation de l'activité} consciente, intentionnelle

La perturbation de l'activité consciente, intentionnelle ${ }^{1}$ est fréquente à la suite d'un traumatisme [16-18] : le patient n'analyse pas les données d'un problème qui lui est posé ; il n'établit pas de schéma ou de programme général de résolution du problème à partir de l'analyse préliminaire de ces données et se limite souvent à donner des réponses impulsives à certains éléments des données qui ont retenu son attention et qu'il a dégagés de l'ensemble ; il éprouve de grandes difficultés à passer d'une opération à une autre; enfin, il ne teste pas les résultats obtenus avec les données initiales du problème (il n'y a pas de signes de cohérence ou d'incohérence entre le résultat de l'action et l'intention initiale).

$\mathrm{m} / \mathrm{s} n^{\circ} 5$ vol. 9, mai 93
Les perturbations du comportement intentionnel se traduisent aussi par des difficultés de communication verbale. Certes, les dérèglements de type aphasique proprement dits sont relativement peu fréquents et peu profonds chez les personnes traumatisées. Par contre, on observe régulièrement une désorganisation linguistique, reflet d'une désorganisation cognitive globale ; elle se manifeste dans le discours par des ruptures, un enchaînement incohérent et l'intrusion de matériel non pertinent [19]. Selon Wiig et coll. [20], une telle description suggère une atteinte de la production divergente (fluence, flexibilité, originalité, élaboration) et de la planification de la production linguistique (ensemble appelé habileté métalinguistique).

\section{Répercussions des séquelles sur l'autonomie sociale et professionnelle}

Des changements importants sont observés dans l'accomplissement des activités de la vie quotidienne (AVQ), des loisirs et du travail. Parmi les séquelles psychologiques les plus fréquentes qui limitent la reprise d'activités, on note une diminution de la résistance à la fatigue mentale, un ralentissement du traitement de l'information, des troubles de l'attention, de la mémoire, du comportement intentionnel dont une perception inadéquate de soi et de l'environnement social, menant à des comportements sociaux peu adaptés ; tous ces facteurs exposent la personne à des blessures accidentelles et freinent sa productivité.

Une activité de la vie quotidienne aussi simple que la préparation d'un repas peut devenir ainsi impossible à

1. Le concept de régulation de l'activité consciente ou comportement intentionnel a été introduit par Luria [16]. Il s'applique à toutes les situations de résolution de problème, y compris celles de la vie quotidienne, et recouvre : (1) la perception de l'environnement extérieur et la perception de soi ; (2) la formulation de buts ou l'identification de besoins ; (3) la planification ou détermination et organisation d'étapes et de moyens nécessaires à l'atteinte du but ou à la satisfaction des besoins; (4) l'exécution du plan d'action et (5) la vérification de sa justesse en ce qui touche le but ou le besoin. 
13. Baddeley A, Harris J, Sunderland A, Watts KP, Wilson BA. Closed head injury and memory. In : Levin HS, Grafman J, Eisenberg HM ed. Neurobehavioral recovery from head injury. NY : Oxford University Press, 1987.

14. Zencius A, Wesolowski M, Burke W, Hough S. Managing hypersexual in braininjured clients. Brain injury 1990, vol. 4, 2 : $175-81$.

15. Sullivan SJ, Richer E, Laurent F. The role of and possibilities for physical conditioning programmes in the rehabilitation of traumatically brain in jured persons. Brain Injury 1990, vol. 4, 4: 407-14.

16. Luria AR. The Working Brain. Penguin Book eds 1973 .

17. Pollens RD, McBratnie BP, Burton PL. Beyond cognition : Executive functions in closed head injury. Cognitive Rehabil 1988, 26-32.

18. Vanier M, Mazaux JM. Troubles de la régulation de l'activité mentale de l'efficience intellectuelle et des fonctions exécutives consécutifs à un traumatisme crânien grave. In : Pélissier, Barat et Mazaux eds. Traumatisme crânien grave et médecine de rééducation. Paris : Masson 166-178, 1991.

19. Hartley LL, Jensen PJ. Narrative and procedural discourse after closed head injury. Brain injury 1991 : vol. 5, 3 : 267-85.

20. Wiig EH, Alexander EW, Secord W. Linguistic competence and level of cognitive functioning in adults with traumatic closed head in jury. In : Whitaker HA ed. Neuropsychological Studies of Nonfocal Brain Damage. New york: Springer-Verlag, 186-201, 1988.

21. Dutil E., Forget A., Vanier M. et Gaudreault C. Development of the ADL Profile : an Evaluation for Adults with Severe Head. In : Injury, Occupational Therapy and Health Care. NY : The Haworth Press, 7-22. 1990.

22. Vogenthaler DR, Smith Jr KR, Goldfader P. Head injury, an empirical study : Describing long-term productivity and independent living outcome. Brain Injury, 1989, vol. 3,4 : 355-68.

23. Piera, JB. Les traumatisés crâniens et le sport. In : Pélissier, Barat et Mazaux (eds) Traumatisme crânien grave et médecine de rééducation. Paris: Masson 166-78, 1991.

24. Prigatano GP. Work, love, and play after brain injury. Bulletin of the Menninger Clinic 1989 ; 53 : 414-31.

25. Thomsen IV. Late outcome of very severe blunt head trauma : a 10-15 year second follow-up. I Neurol, Neurosurg, and Psychiat 1984; 47 : 60-268.
26. Brooks N, McKinlay W, Symington C, Beattie A, Campsie L. Return to work within the first seven years of severe head injury. Brain Injury 1987; 1 : 5-19.

27. Mazaux JM. Réinsertion professionnelle des traumatisés crâniens graves. Réadaptation 1986, 332: 3-9.

28. Wehman P, Kreutzer J, Wood W Morton MV, Sherron P. Supported work model for persons with traumatic brain in jury: Toward job placement and retention. Rehabilitation Counseling Bulletin 1988 ; 31: 298-312.

29. Haffey W, Abrams D. Employment outcomes for participants in a brain injury work reentry program: preliminary findings. J Head Trauma Rehabil 1991; vol. 6 , $3: 24-34$.

30. Moore A, Stambrook M, Peters LC Lubusko A. Family coping and marital adjustment after traumatic brain injury. J Head Trauma Rehabil 1991; vol. 6, 1 : 83-9.

31. Brooks DN, Campsie L, Symongton C Beattie A, McKinlay W. The five year outcome of severe blunt head injury : a relative's view. J Neurol Neurosurg and Psychiat 1986, 49 : 764-70.

32. Godfrey HPD, Knigth RG, Bishara SN. The relationship between skill and familiy problem-solving following very severe closed head injury. Brain injury 1991; vol. 5, 2 : 207-11.

33. Mauss-Clum $\mathbf{N}$, Ryan $\mathbf{M}$. Brain in jury and the family. Joumal of Neurosurgical Nursing $1987 ; 13$ : 165-9.

34. Réseau International CIDIH. Le processus de production des handicaps. Comment utiliser le modèle conceptuel-Exemples. Société canadiennne de la CIDIH 1991.

35. Levin $\mathrm{HS}$ et coll. The neurobehavioural rating scale : assessment of the behavioural sequelae of head in jury by the clinician. J Neurol, Neurosurg, Psychiat 1987; 50 183-93.

36. Truelle JL, Brooks DN, Traumatismes crâniens : un document européen d'évaluation. In : Pélissier, Barat et Mazaux eds. Traumatisme crânien grave et médecine de rééducation. Paris : Masson 166-178, 1991.

37. Vanier M., Lambert J, Dutil E, Kemp F, Sullivan J, Forget A, Provost J, Boulanger YL, Drouin G. Evaluation globale et prospective des personnes traumatisées crânio-encéphaliques. IIe Congrès International sur l'Évaluation Mesurable en Médecine de Réadaptation, Montréal, Octobre 1992 accomplir [21] : la personne ne peut clairement identifier ses goûts ou sa faim ; elle est incapable d'élaborer un menu en tenant compte de ce qu'elle peut faire ; en outre, elle a besoin qu'on l'incite à entreprendre la tâche, elle en ordonne mal les étapes de réalisation et risque de l'exécuter de façon dangereuse ; elle l'interrompt s'il manque un élément ou ne la complète pas faute d'identifier ou de corriger ses erreurs. La fatigue et la lenteur accentuent ces difficultés et peuvent conduire la personne à abandonner la tâche en cours d'exécution. Même si elle n'est affectée d'aucun trouble sensorimoteur significatif, la personne victime d'un traumatisme crânio-encéphalique grave ne manifeste bien souvent qu'un intérêt réduit et n'accorde qu'une faible participation aux activités de loisirs individuelles et sociales [22, 23]. Le cercle de ses amis et de ses relations se rétrécit et elle contracte alors une forte dépendance à l'égard de sa famille. Elle se confine à un état de grande passivité. Or, sur le plan thérapeutique, on sait que les activités de loisir jouent un rôle très important : elles constituent un moyen pour la victime de développer ses ressources personnelles (ses talents, ses attitudes, ses intérêts et son habileté à répondre aux influences de son milicu, contribuant ainsi au développement de l'estime de soi et de l'identité personnelle très diminuées [24] mais pourtant indispensables à une reprise d'activités professionnelles.

En ce qui concerne les activités professionnelles, les facteurs les plus liés à la réinsertion au travail semblent être les indices de gravité de l'atteinte crânio-encéphalique, le statut professionnel pré-traumatique et le temps écoulé depuis l'accident. Seulement $35 \%$ des personnes reprennent le travail au cours des deux années qui suivent l'accident $[25,26]$ et plusieurs d'entre elles subissent un reclassement professionnel. L'abandon des activités de travail contribue à augmenter la perte de motivation et d'estime de soi déjà si importante [27]. Pourtant, il semble exister un potentiel de réinsertion chez une proportion importante des traumatisés graves; les programmes de rééducation, les stages préparatoires à 
l'emploi et le soutien en milieu de travail sont des facteurs favorisant la réinsertion au travail de ces personnes [28, 29].

Sur le plan familial, les séquelles et, souvent, le séjour prolongé en centre de soins, entraînent des changements dans le partage des rôles et responsabilités au sein de la famille. Le conjoint pourra faire face à des difficultés conjugales dans les sphères affective, sexuelle, financière, des difficultés aussi dans l'éducation des enfants et les perspectives d'avenir [30]. Les tensions prolongées subies à la suite du traumatisme menacent l'équilibre même des membres de la famille et la stabilité du système familial [ 31 , 32]. Les séquelles ont aussi des répercussions sur le réseau social de la famille, un isolement social graduel ayant tendance à se développer [33]. Les facteurs socio-culturels et écologiques sous-tendant l'organisation d'une société [34] peuvent constituer des obstacles à la réinsertion sociale et professionnelle et créer ainsi une situation de handicap. Les premiers peuvent s'illustrer par une attitude erronée de la société face aux difficultés souvent non visibles de la personne, une insuffisance des services destinés à la personne traumatisée et à sa famille, une grande précarité des ressources communautaires et un taux de chômage élevé pour les personnes traumatisées aptes à retourner au travail ; les seconds s'illustrent par des conditions géographiques défavorables, l'inaccessibilité des lieux (par exemple, des lieux publics, du milieu de travail), une technologie inadaptée.

\section{Conclusion}

Ainsi, les études longitudinales auprès des personnes ayant subi un traumatisme crânien grave soulignent le large éventail des séquelles psychologiques et leurs répercussions à très long terme sur la vie sociale et professionnelle de la victime et de sa famille.

Il apparaît donc primordial qu'un bilan complet des séquelles et de leurs répercussions soit effectué rapidement après le traumatisme et repris périodiquement durant les premières années post-trauma; il permet ainsi d'orienter les interventions thérapeu- tiques de façon à rendre le plus efficace possible la réadaptation et la réinsertion communautaire de la victime ainsi que de développer des interventions appropriées auprès de la famille. Toutefois, un tel bilan, basé sur la performance à des tests objectif $s$, est très exigeant en temps et en effort pour la victime et il peut s'avérer impossible à réaliser durant les premiers mois ou même la première année à cause de la gravité de l'atteinte, de la grande fatigabilité et de la faible motivation de la victime. De plus, il n'est pas toujours possible de le réaliser dans certains milieux de soins, faute de temps ou de ressources.

Il s'avère donc nécessaire de procéder à une évaluation par étapes. Il s'agirait d'abord d'entreprendre un dépistage de l'ensemble des caractéristiques pré - et post-traumatiques pertinentes puis, au besoin, de procéder à une évaluation détaillée à l'aide de tests objectifs, afin de confirmer ou d'infirmer la présence des troubles dépistés et d'en évaluer la gravité. Les entrevues structurées ainsi que les grilles d'observation du comportement font partie des meilleurs moyens pour effectuer le dépistage des incapacités et des handicaps ; elles permettent de recueillir rapidement un vaste éventail d'informations. Leur validité et leur fiabilité sont, bien sûr, très liées à l'expérience de l'examinateur. Les grilles d'observation, encore peu utilisées, permettent aussi de fournir des informations sur les capacités d'adaptation des personnes lorsqu'elles se trouvent dans leur entourage familier.

Les informations appropriées à recueillir et à transmettre à des intervenants de diverses disciplines durant plusieurs années après le traumatisme sont innombrables. Pour cette raison, les intervenants auront fort probablement de plus en plus recours à des systèmes d'évaluation globale et longitudinale [36, 37]. L'informatisation de tels systèmes permettra de gérer efficacement l'importante somme de données relatives à l'évolution de la victime pendant toutes les phases post-traumatiques, dans les différents milieux d'interventions qu'elle sera amenée à fréquenter. De tels systèmes permettront un meilleur suivi et, par conséquent, auront des retombées importantes sur l'évaluation des programmes d'interventions et le développement de nouveaux programmes appropriés [37].

En ce qui concerne ces interventions, les principes maintenant mis de l'avant dans un grand nombre de milieux de soins œuvrant auprès de cette clientèle consistent en une prise en charge précoce de la victime selon une approche individualisée et globale (plutôt que morcelée) par une équipe multidisciplinaire d'intervenants, en une continuité de services tout au long du processus de récupération neurologique - réadaptation - réinsertion communautaire et enfin en un support à la famille [2].

Les jeunes forment la grande majorité des victimes de traumatismes crâniens graves. Faute de soins appropriés, ils risquent de développer des handicaps permanents diminuant considérablement leur qualité de vie et celle de leurs proches pour de très nombreuses années. Il est donc important que se poursuivent les travaux visant une meilleure compréhension et une meilleure évaluation des séquelles et de leurs répercussions ainsi que ceux visant le développement d'interventions cliniques et communautaires appropriées

\section{Summary}

Psychological consequences of severe cranial trauma and repercussions on social and professional autonomy of patients

This paper identifies the major psychological long term sequelae observed following a severe traumatic head injury and discusses their impact on a range of functional activities. In addition, the possibility of a computerized evaluation system is presented as are strategies for therapeutic interventions.

\section{TIRÉS A PART}

M. Vanier. 